Proyecciones

Vol. 21, No 1, pp. 97-108, May 2002.

Universidad Católica del Norte

Antofagasta - Chile

\title{
ON THE VOLUMETRIC ENTROPY IN THE NON COMPACT CASE
}

\author{
ANDRÉS NAVAS \\ École Normale Supérieure de Lyon, France
}

\begin{abstract}
We give an example of a non compact riemannian manifold with finite volume for which the limit corresponding to the classical definition of the volumetric entropy does not exist. This confirms the fact that in the non compact finite volume case, the natural definition is given by the critical exponent of the mean growth rate for the volume on the riemannian covering.
\end{abstract}

Subject classification AMS 2000 : Primary 37A35; Secondary : 37D40, 53C24.

Keywords : Entropy, volume growth. 


\section{Introduction}

Let $(X, g)$ be an $n$-dimensional riemannian manifold, and let $(\tilde{X}, \tilde{g})$ be the riemannian covering. Let us fix a base point $x \in \tilde{X}$ and consider the limit values

$$
\begin{aligned}
& h^{+}(g)=\limsup _{R \rightarrow \infty} \frac{\log \left(\operatorname{vol} \tilde{B}_{g}(x, R)\right)}{R}, \\
& h^{-}(g)=\liminf _{R \rightarrow \infty} \frac{\log \left(\operatorname{vol} \tilde{B}_{g}(x, R)\right)}{R},
\end{aligned}
$$

where $\tilde{B}_{g}(x, R)$ is the ball of radius $R$ and center $x$ in $\tilde{X}$ and vol $\tilde{B}_{g}(x, R)$ its volume (respect to the metric $\tilde{g}$ ). These limits do not depend on the point $x$. We call them the upper and lower volumetric entropy respectively.

If $X$ is compact, or more generally if $(\tilde{X}, \tilde{g})$ has a co-compact subgroup of isometries, then one has $h^{+}(g)=h^{-}(g)$, and this value is called the volumetric entropy of $(X, g)$ and is simply denoted by $h(g)$ (see [Ma] or [Pa]). Let us remark that, for $\lambda>0$, one has $h(\lambda g)=\frac{h(g)}{\sqrt{\lambda}}$, and then the value of $(h(g))^{n} \operatorname{vol}(X, g)$ is invariant by homotheties.

In the compact case, volumetric entropy appears in a natural way in the study of many di- fferent problems. For example, a classical result says that if the volume is normalized, then the volumetric entropy is a lower bound for the topological entropy of the geodesic flow on the unit tangent bundle, and one has the equality in the negative curvature case (see [Ma]). In a slightly different context, M.Gromov's minimal entropy conjecture, proved in [BCG], says that for oriented hyperbolic manifolds of dimension $\geq 3$, the value of $(h(g))^{n} \operatorname{vol}(X, g)$ is minimal only for metrics that are scalar multiples of the hyperbolic one. Among other things, this result gives a positive answer to the minimal volume conjecture (see $[\mathrm{Gr}]$ ), and also gives a beautiful proof of the classical Mostow's rigidity theorem in the compact case.

It is natural to think that this kind of results may be extended to the non compact finite volume case. This is well known for Mostow's theorem, and for the minimal entropy conjecture one has a partial positive answer (see $[\mathrm{CF}]$ ). For the minimal volume conjecture, see $[\mathrm{CF}]$ and $[\mathrm{Bes}]$. 
One of the problems that appears in the case of non compact finite volume manifolds is to give a good definition for the volumetric entropy. Indeed, in this article we give an example of such a manifold for which the upper and lower volumetric entropies are different (see section $\S 1$ ). In this way we confirm the fact that in the non compact finite volume case, the natural definition is given by the critical exponent of the mean growth rate for the volume on the riemannian covering (see section $\S 2$ ).

\section{An example}

The motivation of the following example is the fact that for the real hyperbolic metric $g_{\text {hyp }}$ of dimension $n$ one has

$$
\operatorname{vol}\left(\tilde{B}_{\lambda^{2} g_{h y p}}(x, R)\right)=v_{n-1} \int_{0}^{R} \lambda\left(\sinh \left(\frac{r}{\lambda}\right)\right)^{n-1} d r
$$

where $\lambda>0$ and $v_{n-1}$ is the volume of the euclidian sphere of dimension $n-1$. So, the idea is to consider a manifold for which the curvature varies between two negatives parameters, and in this way to obtain a variation for the exponential growth rate of vol $\tilde{B}_{g}(x, R)$.

The manifold $X$ that we will construct is homeomorphic to a twodimensional cylinder (though, the construction can be done in any dimension). The riemannian covering $\tilde{X}$ is the real plane endowed with the conformal metric given by

$$
\tilde{g}(x)(u, v)=\frac{\exp \left(f\left(x_{2}\right)\right)}{x_{2}^{2}}(u, v)_{e u c l}
$$

where $x=\left(x_{1}, x_{2}\right) \in \mathbf{R}^{2},(\cdot, \cdot)_{\text {eucl }}$ is the euclidian metric and $f$ : $\mathbf{R}-\{0\} \rightarrow \mathbf{R}, \frac{\exp \left(f\left(x_{2}\right)\right)}{x_{2}^{2}}: \mathbf{R} \rightarrow \mathbf{R}^{+}$are smooth functions (to be defined). The manifold $X$ is then the quotient $\tilde{X} / \Gamma$, where $\Gamma$ is the group generated by the isometry $\left(x_{1}, x_{2}\right) \rightarrow\left(x_{1}+1, x_{2}\right)$ (see picture 1 ). Let us remark that for a metric obtained by this method, the vertical lines $x=$ constant are minimal geodesics in $\tilde{X}$.

The construction of the function $f$ will be carried out in several steps. 
We start by fixing two real numbers $a>1$ and $b>1$ such that $\frac{a}{4}=4 b$. In the interval $(-2,2)$ we take $f$ in such a way that $\frac{\exp (f(t))}{t^{2}}=1$ for $t \in(-1,1)$ and $\exp (f(t))=\frac{1}{a^{2}}$ for $t \in\left(-2,-\frac{3}{2}\right) \cup\left(\frac{3}{2}, 2\right)$.

We will construct inductively a sequence of real numbers

$$
2=a_{0}<a_{1}<b_{0}<b_{1}<a_{2}<a_{3}<b_{2}<b_{3}<\ldots,
$$

and we will define the function $f$ on each interval defined by this sequence. We will also consider a sequence of points $P_{k}$ defined by $P_{4 k}=$ $\left(0, a_{2 k}\right), P_{4 k+1}=\left(0, a_{2 k+1}\right), P_{4 k+2}=\left(0, b_{2 k}\right)$ and $P_{4 k+3}=\left(0, b_{2 k+1}\right)$. The origin $(0,0)$ will be our base point, and we will denote it simply by $O$. We will use some terms as ball, distance, radius and diameter, always considered respect to the metric $\tilde{g}$. Let us remark that in some steps of the construction we will use the fact that, for any metric of the form (2.2), the distance between the points $O$ and $P_{k}$ depends only on the values of $f$ on the segment of the geodesic $x=0$ between $O$ and $P_{k}$.

(i) From $a_{2 k}$ to $a_{2 k+1}$.

We define $\exp (f(t))=\frac{1}{a^{2}}$ for $t \in\left(-a_{2 k+1},-a_{2 k}\right) \cup\left(a_{2 k}, a_{2 k+1}\right)$, where $a_{2 k+1}$ is such that

$$
\begin{gathered}
\quad v_{1} \int_{0}^{\frac{\operatorname{dist}\left(P_{4 k}, P_{4 k+1}\right)}{2}} \frac{1}{a} \sinh (a r) d r \\
\geq \frac{v_{1}}{2 a^{2}} \exp \left(\frac{a \cdot \operatorname{dist}\left(P_{4 k}, P_{4 k+1}\right)}{2}\right), \\
\operatorname{dist}\left(P_{4 k}, P_{4 k+1}\right)>\operatorname{dist}\left(O, P_{4 k}\right) .
\end{gathered}
$$

It is easy to see that in the ball whose diameter is the segment of the geodesic joining $P_{4 k}$ and $P_{4 k+1}$, the curvature is constant and equal to $-a^{2}$. This ball is contained in the ball $\tilde{B}_{g}\left(O, \operatorname{dist}\left(P_{4 k}, P_{4 k+1}\right)\right)$. We then obtain, from (2.1), (2.3) and (2.4),

$$
\begin{gathered}
\operatorname{vol}\left(\tilde{B}_{g}\left(O, \operatorname{dist}\left(P_{4 k}, P_{4 k+1}\right)\right)\right) \geq \frac{v_{1}}{2 a^{2}} \exp \left(\frac{a \cdot \operatorname{dist}\left(P_{4 k}, P_{4 k+1}\right)}{2}\right) \\
\geq \frac{v_{1}}{2 a^{2}} \exp \left(\frac{a \cdot \operatorname{dist}\left(P_{4 k}, P_{4 k+1}\right)}{4}\right) .
\end{gathered}
$$

By taking $R_{2 k}=\operatorname{dist}\left(P_{4 k}, P_{4 k+1}\right)$ we obtain

$$
\frac{\log \left(\operatorname{vol} \tilde{B}_{g}\left(O, R_{2 k}\right)\right)}{R_{2 k}} \geq \frac{\log \left(\frac{v_{1}}{2 a^{2}}\right)}{R_{2 k}}+\frac{a}{4} .
$$


(ii) From $a_{2 k+1}$ to $b_{2 k}$.

With the purpose of remaining in negative curvature we will define the points $b_{2 k}^{(i)}, i=0, \cdots, 6$, as follows. Let $\varepsilon>0$ be such that $6(1-\varepsilon)=$ $\log \left(\frac{1}{a^{2}}\right)=\log (256)$. Let us consider a smooth function $h: \mathbf{R} \rightarrow \mathbf{R}$ such that $h(t)=0$ for $t \leq 0, h(t)=1-\varepsilon$ for $t \geq 1$ and $\inf \left(h^{\prime \prime}\right)>$ -1 . We set $b_{2 k}^{(0)}=a_{2 k+1}$, and recursively we define $b_{2 k}^{(i+1)}$ in such a way that $\left(\frac{b_{2 k}^{(i+1)}-b_{2 k}^{(i)}}{b_{2 k}^{(i+1)}}\right)^{2} \geq-\frac{\inf \left(h^{\prime \prime}\right)}{2}$. Then define $f(t)=\log \left(\frac{1}{a^{2}}\right)+$ $i(1-\varepsilon)+h\left(\frac{t-b_{2 k}^{(i)}}{b_{2 k}^{(i+1)}-b_{2 k}^{(i)}}\right)$ for $t \in\left[b_{2 k}^{(i)}, b_{2 k}^{(i+1)}\right]$, and $f(t)=f(-t)$ for $t \in\left[-b_{2 k}^{(i+1)},-b_{2 k}^{(i)}\right]$. Finally, we set $b_{2 k}=b_{2 k}^{(6)}$.

(iii) From $b_{2 k}$ to $b_{2 k+1}$.

We set $\exp (f(t))=\frac{1}{b^{2}}$ for $t \in\left[-b_{2 k+1},-b_{2 k}\right] \cup\left[b_{2 k}, b_{2 k+1}\right]$, where $b_{2 k+1}$ is a parameter to be defined. We will use an auxiliary point $P_{4 k+3}^{\prime}=\left(0, b_{2 k+1}\right)$, which is also to be defined.

Let us define $R_{2 k+1}=\operatorname{dist}\left(O, P_{4 k+3}\right)$. For $x \in \tilde{B}_{g}\left(O, R_{2 k+1}\right)$ let us take a minimal geodesic

$$
\gamma(s)=\left(\gamma_{1}(s), \gamma_{2}(s)\right)
$$

joining $O$ and $x$. We consider the times $s_{i, j}, j=1,2$, that satisfy the following conditions:

$$
\begin{aligned}
& \text { if } s \in\left[s_{i, 1}, s_{i, 2}\right] \text { then } \gamma_{2}(s) \leq b_{2 k} \text {, } \\
& \text { if } s \in\left[s_{i, 2}, s_{i+1,1}\right] \text { then } \gamma_{2}(s) \geq b_{2 k} .
\end{aligned}
$$

We set $l_{i, 1}=\log \left(\left.\gamma\right|_{\left[s_{i, 1}, s_{i, 2}\right]}\right)$ and $l_{i, 2}=\log \left(\left.\gamma\right|_{\left[s_{i, 1}, s_{i, 2}\right]}\right)$. We will obtain some a-priori estimates of $\left|s_{i, 2}-s_{i, 1}\right|$ and $\left|s_{i+1}-s_{i, 2}\right|$ with respect to $R_{2 k+1}$.

First of all, let us remark that there exists a constant $\tilde{C}_{k}>0$ satisfying

$$
(u, v)_{\text {eucl }} \geq \tilde{g}(y)(u, v) \geq \frac{(u, v)_{\text {eucl }}}{\tilde{C}_{k}}
$$

for all $y=\left(y_{1}, y_{2}\right)$ such that $y_{2} \leq b_{2 k}$. We then have $\left|s_{i, 2}-s_{i, 1}\right| \leq \tilde{C}_{k} \cdot l_{i, 1}$. 
On the other hand, a little of hyperbolic geometry shows that (see [Bea], theorem 7.2)

$$
\sinh \left(\frac{b \cdot l_{i, 2}}{2}\right)=\frac{\left|s_{i+1,1}-s_{i, 2}\right|}{2 b_{2 k}},
$$

and then

$$
\left|s_{i+1,1}-s_{i, 2}\right|=2 b_{2 k} \cdot \sinh \left(\frac{b \cdot l_{i, 2}}{2}\right) \text {. }
$$

Therefore,

$$
\begin{aligned}
\sum_{i}\left|s_{i, 2}-s_{i, 1}\right|+\left|s_{i+1,1}-s_{i, 2}\right| & \leq \tilde{C}_{k} \sum_{i} l_{i, 1}+2 b_{2 k} \sum_{i} \sinh \left(\frac{b \cdot l_{i, 2}}{2}\right) \\
& \leq \tilde{C}_{k} \operatorname{dist}(O, x)+b_{2 k} \sum_{i} \exp \left(\frac{b \cdot l_{i, 2}}{2}\right) \\
& \leq \tilde{C}_{k} \operatorname{dist}(O, x)+b_{2 k} \exp \left(\sum_{i} \frac{b \cdot l_{i, 2}}{2}\right) \\
& \leq \tilde{C}_{k} \operatorname{dist}(O, x)+ \\
& b_{2 k} \exp \left(\frac{b \cdot \operatorname{dist}(O, x)}{2}\right) \\
\leq & C_{k}^{\prime} \exp \left(\frac{b \cdot \operatorname{dist}(O, x)}{2}\right) \\
\leq & C_{k}^{\prime} \exp \left(\frac{b \cdot R_{2 k+1}}{2}\right),
\end{aligned}
$$

where $C_{k}^{\prime}$ is independent of $x$ and $b_{2 k+1}^{\prime}$.

Let $C_{k}$ be a constant such that

$$
2 C_{k} b_{2 k} \exp \left(\frac{b \cdot R}{2}\right)+\frac{2 v_{1}}{b^{2}} \exp (2 b \cdot R) \leq C_{k} \exp (3 b \cdot R)
$$

for all $R \in \mathbf{R}$. We fix $b_{2 k+1}$ and $b_{2 k+1}$ once and for all in such a way that

$$
\begin{gathered}
R_{2 k+1} \geq \operatorname{dist}\left(P_{4 k}, P_{4 k+1}\right) \quad \text { et } \operatorname{dist}\left(P_{4 k+3}, P_{4 k+3}\right) \geq R_{2 k+1} \\
\frac{\log \left(C_{k}\right)}{R_{2 k+1}} \leq \frac{1}{k}
\end{gathered}
$$


It is not difficult to see that in the set $\tilde{B}_{g}\left(O, R_{2 k+1}\right) \cap\left\{x:\left|x_{2}\right|>\right.$ $\left.b_{2 k}\right\}$ the curvature is constant and equal to $-b^{2}$. A simple triangular inequality shows that

$$
\begin{aligned}
& \operatorname{vol}\left(\tilde{B}_{g}\left(O, R_{2 k+1}\right) \cap\left\{x: x_{2}>b_{2 k}\right\}\right) \\
& \leq 2 v_{1} \int_{0}^{R_{2 k+1}+\operatorname{dist}\left(P_{4 k}, P_{4 k+1}\right)} \frac{\sinh (b \cdot r)}{b} d r .
\end{aligned}
$$

We then obtain, from (2.6) and (2.10),

$$
\begin{aligned}
& \text { vol }\left(\tilde{B}_{g}\left(O, R_{2 k+1}\right)\right) \leq 2 C_{k} b_{2 k} \exp \left(\frac{b \cdot R_{2 k+1}}{2}\right) \\
& +\frac{2 v_{1}}{b^{2}} \exp \left(b \cdot\left(R_{2 k+1}+\operatorname{dist}\left(P_{4 k}, P_{4 k+1}\right)\right)\right) .
\end{aligned}
$$

From this inequality, from (2.7) and (2.9), we finally obtain

$$
\frac{\log \left(\operatorname{vol} \tilde{B}_{g}\left(O, R_{2 k+1}\right)\right)}{R_{2 k+1}} \leq \frac{\log \left(C_{k}\right)}{R_{2 k+1}}+3 b \leq \frac{1}{k}+3 b
$$

(iv) From $b_{2 k+1}$ to $a_{2 k+2}$.

We proceed in an analogous way to (ii). We set $a_{2 k+2}^{(0)}=b_{2 k+1}$, and we define recursively $a_{2 k+2}^{(i+1)}$ in such a way that $\left(\frac{a_{2 k+2}^{(i+1)}-a_{2 k+2}^{(i)}}{a_{2 k+2}^{(i+1)}}\right)^{2} \geq$ $-\frac{\inf \left(h^{\prime \prime}\right)}{2}, i=0, \cdots, 5$. For $t \in\left[a_{2 k+2}^{(i)}, a_{2 k+2}^{(i+1)}\right]$ set $f(t)=\log \left(\frac{1}{b^{2}}\right)-i(1-$ $\varepsilon)-h\left(\frac{t-a_{2 k+2}^{(i)}}{a_{2 k+2}^{(i+1)}-a_{2 k+2}^{(i)}}\right)$, and set $f(t)=f(-t)$ for $t \in\left[-a_{2 k+2}^{(i+1)},-a_{2 k+2}^{(i)}\right]$.

Finally, we define $a_{2 k+2}=a_{2 k+2}^{(6)}$. 

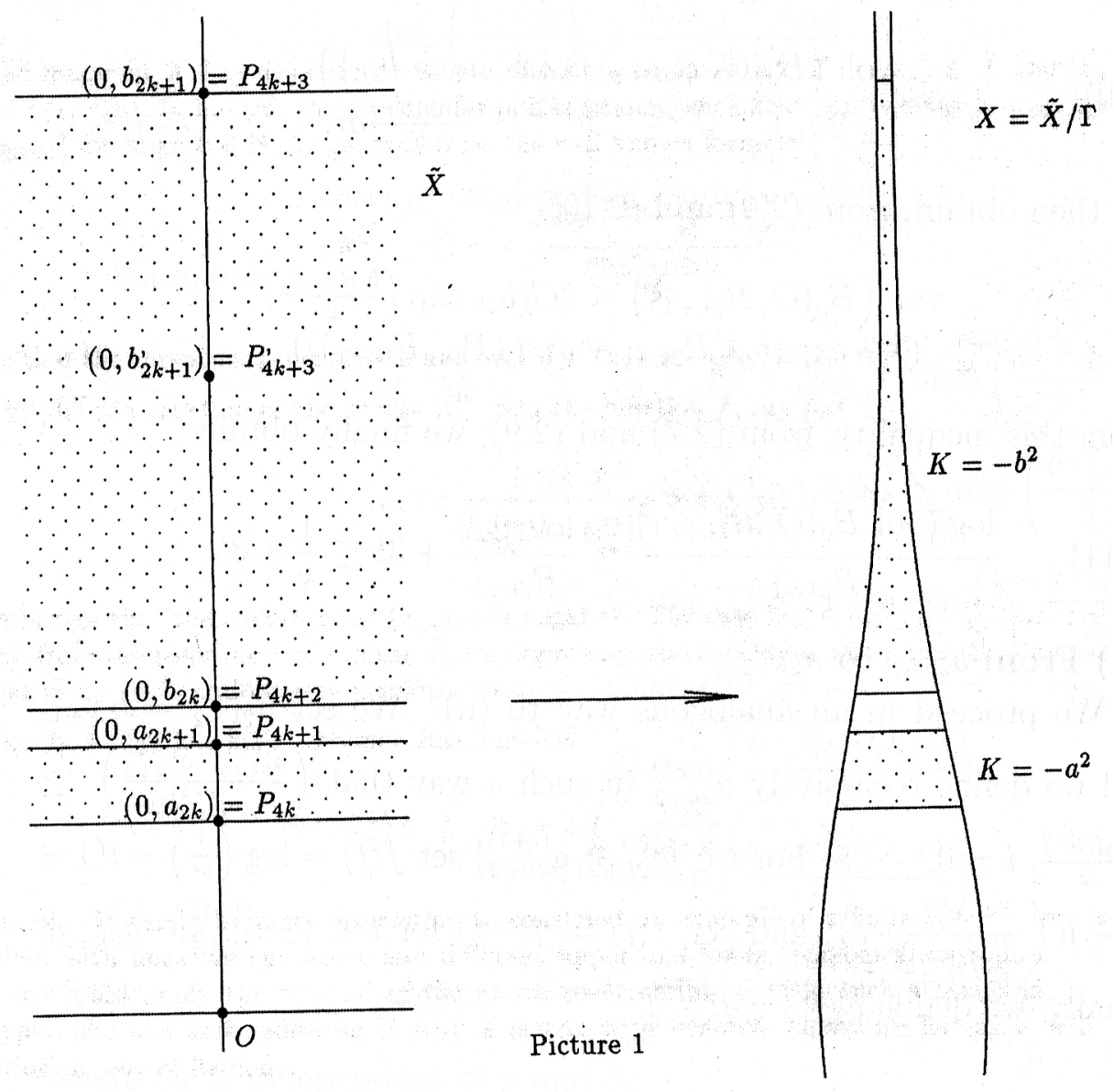
Now we collect some properties of the manifold $X$.

By (2.5) one has $h^{+}(g) \geq \frac{a}{4}$, and from (2.11) we obtain $h^{-}(g) \leq 3 b$. Since $\frac{a}{4}=4 b$, one has $h^{+}(g)>h^{-}(g)$.

To prove that $X$ is a complete manifold, let us consider the compact sets $K_{n}=\pi([-n, n] \times[-n, n])$, where $\pi: \tilde{X} \rightarrow X$ is the covering map. By Hofp-Rinow's theorem, we have to prove that if $\pi\left(\left(x_{n}, y_{n}\right)\right)$ is a sequence in $X$ such that $0 \leq x_{n}<1$ and $\left(x_{n}, y_{n}\right)$ does not belong to $K_{n}$, then $\operatorname{dist}_{X}\left(\pi(O), \pi\left(x_{n}, y_{n}\right)\right)$ goes to infinity as $n \rightarrow \infty$. But this follows directly from the inequality

$$
\operatorname{dist}_{X}\left(\pi(O), \pi\left(x_{n}, y_{n}\right)\right) \geq \operatorname{dist}_{\tilde{X}}\left(O,\left(x_{n}, y_{n}\right)\right) \geq \int_{0}^{n} \frac{d t}{b \cdot t}=\frac{\log (n)}{b} .
$$

The manifold $X$ has negative curvature outside a compact set: if $\left(x_{1}, x_{2}\right) \in \tilde{X}$ and $\left|x_{2}\right| \geq 2$, then $K\left(x_{1}, x_{2}\right)<0$. Indeed, this is clear for points $\left(x_{1}, x_{2}\right)$ such that $\left|x_{2}\right|$ belongs to $\left[a_{2 k}, a_{2 k+1}\right]$ or $\left[b_{2 k}, b_{2 k+1}\right]$ for some $k \in \mathbf{N}$. In general, from the well known formula

$$
K\left(x_{1}, x_{2}\right)=-\frac{\Delta \log \left(\frac{\exp \left(f\left(x_{1}, x_{2}\right)\right)}{x_{2}^{2}}\right)}{\frac{\exp \left(f\left(x_{1}, x_{2}\right)\right)}{x_{2}^{2}}},
$$

we see that the curvature is negative if and only if $f^{\prime \prime}\left(x_{2}\right)>-\frac{2}{\left|x_{2}\right|^{2}}$. If $\left|x_{2}\right| \in\left[b_{2 k}^{(i)}, b_{2 k}^{(i+1)}\right], i=0, \cdots, 5$, then by the construction of the points $b_{2 k}^{(i)}$ and the function $f$, one has

$$
f^{\prime \prime}\left(x_{2}\right) \geq \frac{\inf \left(h^{\prime \prime}\right)}{\left(b_{2 k}^{(i+1)}-b_{2 k}^{(i)}\right)^{2}}>-\frac{2}{\left|x_{2}\right|^{2}},
$$

which proves that the curvature at $\left(x_{1}, x_{2}\right)$ is negative. The case $\left|x_{2}\right| \in\left[a_{2 k+2}^{(i)}, a_{2 k+2}^{(i+1)}\right]$ is analogous. In fact, from the construction and the above arguments one concludes easily that the curvature has a negative upper bound outside a compact set.

Finally, $(X, g)$ has finite volume, since one has

$$
\operatorname{vol}(X, g) \leq \int_{-2}^{2} d x_{2}+2 \int_{2}^{\infty} \frac{d x_{2}}{\left(b x_{2}\right)^{2}}=4+\frac{1}{b^{2}}
$$


Remark. It would be more interesting to construct an example of a finite volume non compact manifold with negative curvature and different upper and lower topological entropies. We think that one could apply the method of the above construction to yield such a manifold, by taking an hyperbolic one and endowing it with a metric with negative curvature having a well chosen variation on one of its ends.

\section{The critical exponent}

Let $(X, g)$ be a riemannian manifold of dimension $n$. We fix a point $x \in \tilde{X}$ and we consider the value of the following integral

$$
I_{s}(x)=\int_{\tilde{X}} \exp (-s \cdot \operatorname{dist}(x, y)) d v_{\tilde{g}}(y) .
$$

Clearly, there exist a parameter $\delta(x) \in[-\infty, \infty]$ such that $I_{s}(x)$ is finite for $s>\delta(x)$ and is equal to infinity for $s<\delta(x)$. It is easy to see that this parameter does not depend on the point $x \in \tilde{X}$. For simplicity, we will denote it by $\delta$.

Proposition. One has the equality $h^{+}(g)=\delta$.

Proof. First, we will prove that $h^{+}(g) \geq \delta$. We can suppose that $h^{+}(g)<\infty$, and then we have to prove that $I_{s}(x)<\infty$ for $s>h^{+}(g)$. By taking $\lambda=\frac{1}{2}\left(s-h^{+}(g)\right)>0$ one has $s-\lambda>h^{+}(g)$. Then, there exist a constant $C=C(x)$ such that for all $r>0$ one has

$$
\text { vol } \tilde{B}_{g}(x, r) \leq C \exp ((s-\lambda) r) \text {. }
$$

This gives

$$
\begin{aligned}
\int_{\tilde{B}_{g}(x, R)} \exp (-s \cdot \operatorname{dist}(x, y)) d v \tilde{g}(y) & =\int_{0}^{R} \exp (-s r) \operatorname{vol} \tilde{S}_{g}(x, r) d r \\
& =\int_{0}^{R} \exp (-s r) \frac{d}{d t}\left(\operatorname{vol} \tilde{B}_{g}(x, r)\right) d r \\
& =s \int_{0}^{R} \exp (-s t) \operatorname{vol} \tilde{B}_{g}(x, r) d r+=
\end{aligned}
$$




$$
\exp (-s R) \operatorname{vol} \tilde{B}_{g}(x, R)
$$

where $\tilde{S}_{g}(x, r)$ is the sphere of center $x$ and radius $r$ in $\tilde{X}$, and vol $\tilde{S}_{g}(x, r)$ is its volume with respect to the $(n-1)$-dimensional induced metric. We then obtain, for $R>0$,

$$
\begin{gathered}
\int_{\tilde{B}_{g}(x, R)} \exp (-s \cdot \operatorname{dist}(x, y)) d v_{\tilde{g}}(y) \leq C s \int_{0}^{R} \exp (-\lambda r) d r \\
+C \exp (-\lambda R) \leq C\left(\frac{s}{\lambda}+1\right)
\end{gathered}
$$

which implies that $h^{+}(g) \geq \delta$.

To prove the opposite inequality, we have to verify that $s+\varepsilon \geq$ $h^{+}(g)$ for all $s>\delta$ and all $\varepsilon>0$. Let us denote by $\mathbf{R}_{\varepsilon}$ the (unbounded) set of positive real numbers $R$ such that

$$
\frac{\log \left(\operatorname{vol} \tilde{B}_{g}(x, R)\right)}{R}>h^{+}(g)-\varepsilon .
$$

For $R \in \mathbf{R}_{\varepsilon}$ we have

$$
\begin{aligned}
\int_{\tilde{X}} \exp (-s \cdot \operatorname{dist}(x, y)) d v_{\tilde{g}}(y) & >\int_{\tilde{B}_{g}(x, R)} \exp (-s \cdot \operatorname{dist}(x, y)) d v_{\tilde{g}}(y) \\
& >\exp (-s R) \operatorname{vol} \tilde{B}_{g}(x, R) \\
& >\exp \left(R\left(h^{+}(g)-s-\varepsilon\right)\right)
\end{aligned}
$$

The left member of this inequality is finite for $s>\delta$ and the set $\mathbf{R}_{\varepsilon}$ is unbounded. Therefore, one has necessarily $h^{+}(g)-s-\varepsilon \leq 0$.

A slight modification of of the first part of the above proof allows to show that $\delta \geq h^{-}(g)$. As a consequence we reobtain in the compact case the well known equality $\delta=h(g)$. In the non compact case, it is natural to take this equality as a definition of volumetric entropy, that is $h(g)=\delta=h^{+}(g)$.

Acknowledgments. It is a pleasure for me to thank G.Courtois for his interest on this work, and I also thank J.Souto for many interesting discussions on this subject. 


\title{
References
}

[Bea] A.BeArdon. The geometry of discrete groups. GTM 91, Springer Verlag (1983).

[Bes] L.Bessières. Sur le volume minimal des variétés ouvertes. Ann. Inst. Fourier (Grenoble) 50 (2000), pp.965-980.

[BCG] G.Besson, G.Courtois \& S.Gallot. Entropies et rigidités des espaces localement symétriques. Geometric and Functional Analysis 5 (1995), pp.731-799.

[CF] C.Connell \& B.FARB. Minimal entropy rigidity for lattices in products of rank one symmetric spaces. Preprint: math.uchicago.edu/ farb/papers.html.

[Gr] M.Gromov. Volume and bounded cohomology. Publ. Math IHES 56, (1981), pp.213-307.

[Ma] A.Manning. Topological entropy for geodesic flows. Ann. Math. 110 (1979), pp.565-573.

[Pa] G.Paternain. Geodesic flows. Birkhäuser (1999).

[1] G. Prasad. Strong rigidity of $Q$-rank 1 lattices. Inv. Math. 21 (1973), pp.255-286.

Received : January, 2002.

\author{
Andrés Navas \\ Unité de Mathématiques Pures et Appliquées, \\ École Normale Supérieure de Lyon \\ UMR 5669 du CNRS \\ 46 allée d'Italie \\ F-69364 Lyon 07 \\ France \\ e-mail: anavas@umpa.ens-lyon.fr
}


